Aim of the study: To assess the efficacy and safety of transcatheter arterial chemoembolization (TACE) plus S-1 for the treatment of Barcelona Clinic Liver Cancer (BCLC) Stage B HCC refractory to TACE.

Material and methods: 26 patients meeting the eligibility criteria were enrolled. TACE was given on day 1 , and S-1 on days 2-15. Tumor assessment was performed one month later according to mRECIST. The primary endpoints were TTP and OS.

Results: Twenty-six patients received 176 TACE interventions in all. Fifteen patients of TACE plus S-1 received a total of 55 cycles of treatment of $\mathrm{S}-1$, with a median of 4 cycles (range, 2-6). The total dose of S-1 was 6165 mg per day, while average was $120 \mathrm{mg}$ (range, 100-125 mg) for 15 patients of TACE plus S-1. Median TTP and OS of TACE plus S-1 were 6 months (95\% $\mathrm{Cl}$ : 4.7-7.3) and 18 months (95\% Cl: 15.3-24.7), respectively, while TACE monotherapy was 4 months $(95 \% \mathrm{Cl}$ : 2.4-5.6) and 13 months (95\% Cl: 9.816.2 ), respectively, and significant differences were detected. Though there were higher DCRs in patients of TACE plus S-1, no significant differences were detected. A total of 612 adverse events occurred during the course of the treatment, 367 in TACE plus S-1 and 245 in TACE mono-therapy. There were significant differences to anorexia and nausea, but they were tolerable. Conclusions: TACE plus S-1 in the present analysis was tolerable and associated with an interesting TTP and OS. TACE plus S-1 may be used as a new treatment method to BCLC Stage B HCC refractory to TACE.

Key words: hepatocellular carcinoma transcatheter arterial chemoembolisation, S-1.

Contemp Oncol (Pozn) 2016; 20 (6): 468-474 DOI: https://doi.org/10.5114/wo.2016.65607

\section{Transcatheter arterial chemoembolisation (TACE) plus S-1 for the treatment of BCLC stage B hepatocellular carcinoma refractory to TACE}

\author{
Wu-Kui Huang ${ }^{1^{*}}$, Shu-Fa Yang ${ }^{1^{*}}$, Li-na You ${ }^{2^{*}}$, Mo Liu ${ }^{1}$, Deng-Yao Liu ${ }^{1}$, \\ Peng Gu${ }^{1}$, Xi-Wen Fan ${ }^{1}$ \\ ${ }^{1}$ Department of Intervention Radiology, the Affiliated Tumour Hospital of Xinjiang \\ Medical University, Urumqi, Xinjiang, China \\ 2Department of Traditional Chinese Medicine, the Fifth Affiliated Hospital of Xinjiang \\ Medical University, Urumqi, Xinjiang, China \\ *These authors contributed equally to this work.
}

\section{Introduction}

Hepatocellular carcinoma (HCC) is one of the most usual cancers worldwide. HCC ranks sixth regarding prevalence and third regarding mortality among malignant tumours [1]. The international recommendations on the management of HCC, presented in 2001 [2], adopted by the American Association for the Study of Liver Diseases (AASLD) in 2005 [3], and recently updated by the European Association for the Study of Liver (EASL) [4, 5], indicate transcatheter arterial chemoembolisation (TACE) as the standard of care for patients with BCLC stage B HCC. This indication is supported basically by several randomised controlled trials (RCT) $[6,7]$ and two meta-analyses $[8,9]$ that demonstrate that TACE improves the overall survival when compared with the best supportive care.

When patients with BCLC stage B HCC fail to profit from TACE, what should be done? So far, there is no standard recommended. Maybe, the answer is sorafenib. To our knowledge, sorafenib is the only systemic thttterapy approved by the United States Food and Drug administration (FDA) for the treatment of unresectable HCC. Two phase III clinical trials have explored the efficacy and safety of sorafenib in patients with advanced HCC [10, 11]. But, sorafenib is not suitable for major Chinese patients due to its high number of adverse reactions. So, what can we do about them?

Based on its potent inhibition of dihydropyrimidine dehydrogenase (DPD), S-1 is expected to be more active than other flouropyrimidines against HCC with higher DPD activity. China is a developing country, and S-1 is available within the content of Chinese medical insurance. Recently, some studies have confirmed the effect of S-1 for HCC. In the study by Furuse, S-1 shows an acceptable toxicity profile and promising antitumour activity for HCC [12]. In addition, in the study by Kim, S-1 and platinum combination chemotherapy showed favourable efficacy and tolerability in advanced HCC [13].

Therefore, we designed this retrospective analysis. The main aim of our analysis was to assess the efficacy and safety of TACE plus S-1 for the treatment of BCLC Stage B hepatocellular carcinoma refractory to TACE.

\section{Material and methods}

\section{Patients}

All patients referred to our tertiary hospital with the diagnosis of BCLC Stage B HCC between August 2012 and April 2015 were included in this retrospective analysis, and they were refractory to TACE. Patients were di- 


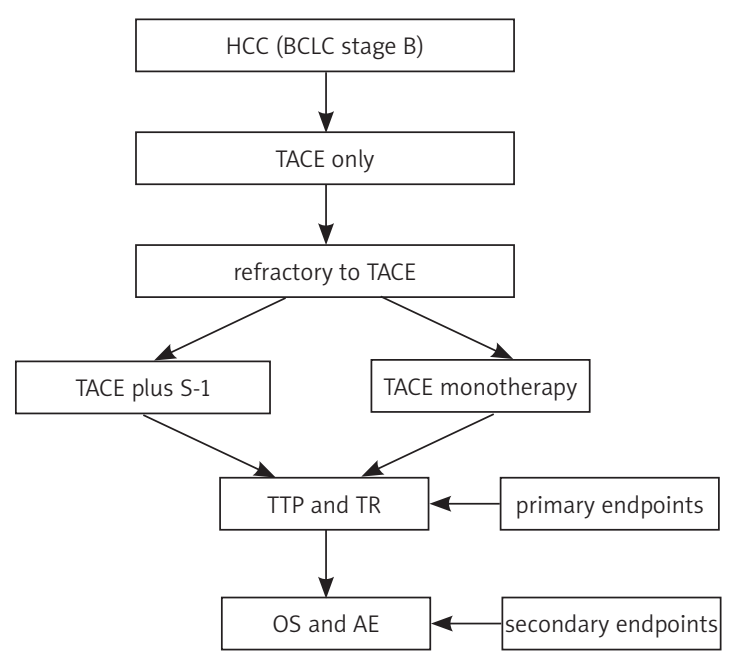

Fig. 1. Flow diagram of the patient selection procedure

vided into two groups: the TACE plus S-1 group and the TACE monotherapy group. The flow diagram of the patient selection procedure can be seen in Fig. 1. Eligible patients had only received TACE therapy before this analysis. Further inclusion criteria were as follows: age $\geq 18$ years, a life expectancy of $\geq 3$ months, Child-Pugh (CP) score of $\leq 8$, at least one measurable lesion, an Eastern Cooperative Oncology Group (ECOG) performance status (PS) score of 0 , absolute neutrophil count $>1,500 / \mathrm{mm}^{3}$, platelet count $>100,000 / \mathrm{mm}^{3}$, haemoglobin $>9 \mathrm{~g} / \mathrm{dl}$, partial thromboplastin time (PTT) within normal limits and international normalised ratio (INR) $<1.5$ upper limit of normal $(U L N)$, a serum creatinine $<1.5 \times$ ULN, total bilirubin (TBil) levels of $\leq 4.5 \mathrm{mg} / \mathrm{dl}$, alanine transaminase (ALT) or aspartate transaminase (AST) $<5 \times U L N$, and alkaline phosphatase $($ ALP) $<4 \times$ ULN.

Written, informed consents was obtained from all patients with HCC prior to each treatment. The analysis protocol complied with all the provisions of the Declaration of Helsinki. This analysis was approved by the Ethics Committee of the Affiliated Tumour Hospital of Xinjiang Medical University, China, and the need for written informed consent was waived since the data were analysed anonymously and retrospectively.

\section{BCLC stage B hepatocellular carcinoma}

The diagnosis of HCC was according to AASLD criteria. A patient was considered to have a confirmed HCC diagnosis when typical hallmark of HCC (hypervascular in the arterial phase with washout in the portal venous or delayed phases) was observed in multi-slice spiral computed tomography (CT) scan or dynamic contrast-enhanced magnetic resonance imaging (MRI) on nodules beyond 1 centimetre $(\mathrm{cm})$ in diameter. Biopsy was reserved for uncertain diagnosis of high-grade dysplastic nodules or discordant results. The BCLC stage B of HCC included CP class $\mathrm{A}$ or $\mathrm{B}$, a single nodule $>5 \mathrm{~cm}$ or multinodular disease ( $>3$ nodules, with at least one lesion $>3 \mathrm{~cm}$ ), good PS (PS 0), and no extrahepatic disease or vascular invasion.

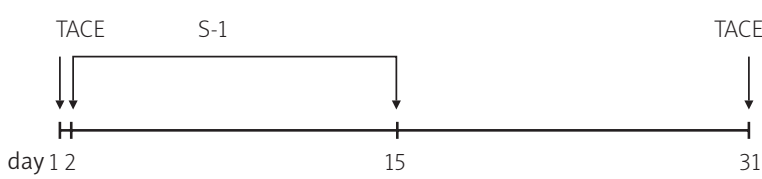

Fig. 2. Treatment cycle schedule

Transcatheter arterial chemoembolization and S-1

One cycle of TACE was 1 month. TACE was performed by selective transarterial chemoembolization in the vessels feeding the tumour using an emulsion of lipiodol (5-20 ml) and pirarubicin (20-60 mg) (with or without infusion chemotherapy with oxaliplatin 50-80 mg), followed by embolisation with absorbable particles of gelatine sponge. One cycle of S-1 was 14 days. Patients of TACE plus S-1 received S-1 30-40 mg/m² bid on day 2 after TACE (day 1) (Fig. 2). Patients of TACE monotherapy received TACE only (day 1). One month after the previous TACE, a multi-slice spiral CT of the upper abdomen and a determination of the a-fetoprotein (AFP) were performed to assess the need of a consecutive TACE. After the first time, TACE intervention was performed on demand. If a follow-up CT scan showed viable tumour in HCC, another course of TACE was scheduled. When no change to viable tumour was seen on the CT, TACE was discontinued no matter which group. If the CT scan revealed new lesions, the patient was evaluated for the feasibility of a new TACE intervention. If new lesions were present in the liver, the patient was treated as having disease progression and received the next course of TACE. If new lesions were seen in extrahepatic sites or major vascular invaded, the patient could receive TACE as well because there was no money to fund the treatment of sorafenib. Patients of TACE plus S-1 choose whether to continue to receive the treatment of S-1 by themselves when there was disease progression. The end of the latest follow-up time was February 2016.

\section{Refractory to transcatheter arterial chemoembolization}

There was no unified definition of "refractory to TACE". In the present analysis, the definition of "refractory to TACE" was regarded as disease progression or a tumour shrinkage rate of $<25 \%$ in hypervascular lesions according to the modified Response Evaluation Criteria in Solid Tumours (mRECIST) after 1-2 months of TACE.

\section{Assessment}

The mRECIST criteria were used to assess lesion size and tumour response. A maximum of two lesions per organ and five lesions in total for one patient, as well as the presence or absence of distant metastases, were evaluated using CT or MRI images at the time of therapeutic assessment. The presence or absence of distant metastatic tumour was recorded in the diagnosis report by assigned radiologists. According to the mRECIST criteria, the target response was in consideration of the change in designated target lesion only. However, overall responses comprehensively were in consideration of changes in target and 
Table 1. Baseline characteristics

\begin{tabular}{|c|c|c|c|}
\hline Variable & $\begin{array}{c}\text { TACE plus } \\
\text { S-1 } \\
(n=15)\end{array}$ & $\begin{array}{c}\text { TACE } \\
\text { monotherapy } \\
(n=11)\end{array}$ & $\begin{array}{c}P \\
\text { value }\end{array}$ \\
\hline $\begin{array}{l}\text { Gender, Male/ } \\
\text { Female }(n)\end{array}$ & $10 / 5$ & $7 / 4$ & 1.000 \\
\hline $\begin{array}{l}\text { Age, year } \\
(\text { mean } \pm S D)\end{array}$ & $61.7 \pm 11.0$ & $62.1 \pm 11.6$ & 0.925 \\
\hline $\begin{array}{l}\text { Tumor size, cm } \\
(\text { mean } \pm \mathrm{SD})\end{array}$ & $7.9 \pm 3.8$ & $7.2 \pm 4.4$ & 0.658 \\
\hline $\begin{array}{l}\text { Tumor number, } \\
\text { median (range) }\end{array}$ & $3(1-10)$ & $4(1-8)$ & 0.854 \\
\hline $\begin{array}{l}\text { Cirrhosis, case } \\
\text { number }(n)\end{array}$ & 10 & 7 & 1.000 \\
\hline $\begin{array}{l}\text { Hepatitis B, case } \\
\text { number }(n)\end{array}$ & 10 & 8 & 1.000 \\
\hline $\begin{array}{l}\text { Hepatitis C, case } \\
\text { number }(n)\end{array}$ & 1 & 0 & NA \\
\hline $\begin{array}{l}\alpha \text {-fetoprotein } \\
>400 \mathrm{ng} / \mathrm{m}(n)\end{array}$ & 8 & 6 & 1.000 \\
\hline $\begin{array}{l}\text { Total bilirubin, } \\
\mu m o l / l \\
(\text { mean } \pm \text { SD) }\end{array}$ & $21.1 \pm 5.4$ & $20.5 \pm 7.2$ & 0.786 \\
\hline $\begin{array}{l}\text { Albumin, g/dl } \\
\text { (mean } \pm S D)\end{array}$ & $34.9 \pm 4.6$ & $35.5 \pm 4.1$ & 0.742 \\
\hline $\begin{array}{l}\text { Alanine } \\
\text { aminotransferase, } \\
\mathrm{U} / \mathrm{I}(\text { mean } \pm \mathrm{SD})\end{array}$ & $46.8 \pm 21.9$ & $44.6 \pm 17.9$ & 0.781 \\
\hline $\begin{array}{l}\text { Platelets, } 10^{3} / \mu \mathrm{l} \\
(\text { mean } \pm \mathrm{SD})\end{array}$ & $97.5 \pm 27.4$ & $96.6 \pm 37.6$ & 0.949 \\
\hline $\begin{array}{l}\text { Prothrombin time, } \\
\text { second } \\
\text { (mean } \pm \mathrm{SD})\end{array}$ & $12.4 \pm 1.9$ & $12.7 \pm 2.1$ & 0.650 \\
\hline $\begin{array}{l}\text { Previous TACE, } \\
\text { median (range) }\end{array}$ & $3(1-5)$ & $3(1-5)$ & 0.808 \\
\hline
\end{tabular}

NA - not available

nontarget lesions, as well as new intrahepatic and extrahepatic lesions.

\section{Study objectives}

The primary endpoints were time to progression (TTP) and overall survival (OS). TTP was defined as the time from the start of treatment of this analysis until criteria for progressive PD were met. OS was measured from the start of treatment of this analysis until date of death or the end of follow-up. Patients who were alive or lost to follow-up were censored at the last date known to be alive. The secondary endpoints were tumour response (TR) and adverse events (AEs). TR consisted of complete response (CR), partial response (PR), stable disease (SD) and progression of disease (PD). Disease control rate (DCR) was defined as the percentage of subjects achieving either a confirmed complete, partial or stable tumour response. AEs were categorised and evaluated according to the American
Common Terminology Criteria for Adverse Events (CTCAE, version 3.0).

\section{Statistical analyses}

We used Statistic Package for the Social Science (SPSS, version 15.0) for all statistical analyses. For all tests of this analysis, a $P$ value $<0.05$ was considered statistically significant. All demographic and clinicopathological data was prospectively collected in a computer database. Continuous data were expressed as mean \pm standard deviation (SD). Differences between continuous data were analysed using $t$ test. Differences between categorical data were analysed using the chi-square test. Survival analysis of TTP and OS were calculated by the Kaplan-Meier method and group results were compared using the log-rank test.

\section{Results}

\section{Baseline characteristics}

A total of 26 patients were screened. Baseline characteristics are summarised in Table 1. Viral hepatitis accounted for $73 \%[(10+8+1) / 26]$ of the underlying causes for HCC, and the majority were hepatitis B. Cirrhosis, mainly caused by viral hepatitis, accounted for $65 \%[(10+7) / 26]$. All patients only received the treatment of TACE, and median number of previous TACE of two groups were 3 (range, 1-5). Compared to the TACE monotherapy, significant differences in the patient characteristics were not observed in variables (Table 1) between the two groups. Because of the low incidence of hepatitis C, statistical inspection was not performed.

\section{Treatment of transcatheter arterial chemoembolization and S-1}

26 patients received 176 TACE interventions in total. There were 101 cycles in 15 patients of TACE plus S-1 and 75 cycles in 11 patients of TACE monotherapy. And for every patient, the mean TACE intervention was 6.8. Patients of TACE plus S-1 received a mean of $8.1 \pm 3.1$ (range, 3-13) TACE interventions, resulting in a mean dose of oxaliplatin $44.9 \pm 36.7 \mathrm{mg}$ and pirarubicin $33.8 \pm 13.0 \mathrm{mg}$ and lipiodol $10.4 \pm 4.1 \mathrm{ml}$. Meanwhile, patients of TACE monotherapy received a mean of $7.5 \pm 2.4$ (range, 4-12) TACE interventions, resulting in a mean dose of oxaliplatin $49.5 \pm 37.3 \mathrm{mg}$ and pirarubicin $37.9 \pm 12.4 \mathrm{mg}$ and lipiodol $11.8 \pm 3.4 \mathrm{ml}$. For S-1, one cycle was 14 days. 15 patients of TACE plus S-1received a total of 55 cycles of treatment of S-1, with a median of 4 cycles (range, 2-6). The total dose of S-1 was $6165 \mathrm{mg}$ per day, while the average was $120 \mathrm{mg}$ (range, 100-125) for 15 patients of TACE plus S-1.

\section{Time to progression}

Calculations of TTP were based on 26 patients in two groups of TACE plus S-1 and TACE monotherapy according to mRECIST criteria. Median TTP of TACE plus S-1 was 6 months [95\% confidence interval (CI): 4.7-7.3] while TACE monotherapy was 4 months ( $95 \% \mathrm{Cl}$ : $2.4-5.6)$. And there was a significant difference (Table 2). The Kaplan-Meier survival curves of TTP can be seen in Fig. 3. 
Table 2. TTP and OS

\begin{tabular}{lcccc} 
Variable & TACE plus S-1 & TACE mono-therapy & $\chi^{2}$ & $P$ value \\
& month, median (range) & month, median (range) & & 0.015 \\
TTP & $6(1-13)$ & $4(1-5)$ & 4.220 & 0.040 \\
\hline OS & $18(8-31)$ & $13(7-21)$ & 5.918 &
\end{tabular}

\section{Overall survival}

Calculations of OS were based on 26 patients in two groups according to mRECIST criteria. Median OS of TACE plus S-1 was 18 months (95\% Cl: 15.3-24.7) while TACE monotherapy was 13 months (95\% Cl: 9.8-16.2). Maybe there was an exciting result, but no significant difference was detected (Table 2). The Kaplan-Meier survival curves of OS can be seen in Fig. 4. After failling to respond to therapy of TACE plus S-1, one patient chose the treatment of sorafenib and another received combination chemotherapy with cisplatin and capecitabine. Three patients were lost to follow-up because the phone numbers recorded in the medical records were wrong or changed. All patients were followed up for at least 10 months.

\section{Tumour response}

There were 1 CR, 6 PR, 5 SD out of 15 patients of TACE plus S-1 and O CR, 2 PR, 5 SD out of 11 patients of TACE monotherapy, respectively. The DCRs of TACE plus S-1 and TACE monotherapy were 80\% (12/15) and 63.6\% (7/11), respectively. Although there were higher DCRs in patients of TACE plus S-1, no significant differences were detected $(p$ value $=0.864)$

\section{Adverse events}

Adverse events were evaluable in 26 patients and 176 treatment cycles of TACE with or without S-1. A total of 612 AEs occurred during the course of the treatment, 367 in TACE plus S-1 and 245 in TACE monotherapy. The usual AEs of the clinic are shown in Table 3. The highest incidence of AE was abdominal pain, which $70.3 \%$ (71 out of 101) in TACE plus S-1 and $69.3 \%$ (52 out of 75 ) in TACE monotherapy. Because of the low incidence, statistical inspection was not performed for diarrhoea, hand-foot syndrome, neuropathy, and bleeding. There were no significant difference to AEs except for anorexia and nausea, which may be due to S-1. Anorexia and nausea were acceptable because they could reduce or disappear after supportive therapy.

\section{Discussion}

Hepatocellular carcinoma (HCC) is one of the most common digestive system malignancies in the world. According to the World Health Organisation (WHO), HCC accounted for a total of 619,000 deaths in 2002, representing $2.5 \%$ of the deaths worldwide [14]. Incidence rates in endemic countries such as East Asia and subequatorial Africa amount to 15-40/100,000, whereas Western countries show an incidence of HCC below 5/1,000,000, although the HCC burden is increasing [15]. For patients

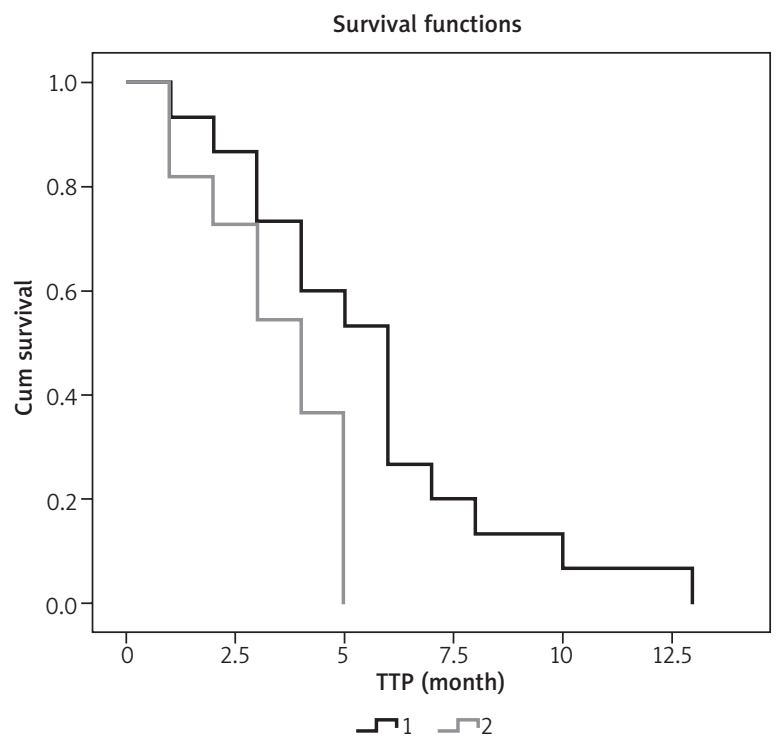

Fig. 3. Time to progression (TTP) curves of TACE plus S-1 group and TACE monotherapy group

with BCLC stage B HCC who are not candidates for resection, TACE is safe [16] and the main therapeutic option [17]. Many Chinese patients with BCLC stage B HCC had already been treated with TACE, and also obtained the certain effect. However, due to the resistance of tumours, there were probable refractory to TACE after several cycles. In other words, tumours were no-responsive to TACE again. Patients refractory to TACE may have poor prognosis. Currently, for patients with BCLC stage B HCC refractory to TACE, there are no criteria used for how to select treatment to optimise the benefit. Sorafenib maybe recommended as the standard treatment of BCLC Stage HCC refractory to TACE. However, sorafenib is a self-paying drug in China. It costs $¥ 150,000$ (renminbi, RMB) for each patient. In a developing countries, most patients cannot afford this cost.

Systematic chemotherapy had been recognised as a palliative treatment option for patients with advanced HCC [18], such as combination chemotherapy of oxaliplatin and S-1 [19]. S-1 is a novel oral 5-fluoro-2, 4 (1 h, 3 h) pyrimidinedione (5-FU) analogue, which contains tegafur and two biochemical modulators for 5-FU. Tegafur is a metabolically activated prodrug of 5-FU, and it is effective as adjuvant chemotherapy after TACE [20]. 5-Chloro-2, 4-dihydroxypyridine can enhance the pharmacological actions of 5-FU by inhibiting its degradation by dihydropyrimidine dehydrogenase (DPD). Potassium oxonate is localised in the mucosal cells of the gastrointestinal tract after oral administration. By means of suppressing the 
Table 3. Adverse events (AE) $[n(\%)]$

\begin{tabular}{|c|c|c|c|}
\hline $\mathrm{AE}$ & $\begin{array}{l}\text { TACE plus S-1 } \\
\quad(n=101)\end{array}$ & $\begin{array}{c}\text { TACE } \\
\text { mono-therapy } \\
(n=75)\end{array}$ & $\begin{array}{c}P \\
\text { value }\end{array}$ \\
\hline Abdominal pain & $71(70.3)$ & $52(69.3)$ & 0.890 \\
\hline Anorexia & $25(24.8)$ & $9(12.0)$ & 0.034 \\
\hline Nausea & $40(39.6)$ & $17(22.7)$ & 0.018 \\
\hline Diarrhea & $0(0.0)$ & $1(1.3)$ & NA \\
\hline Hyperbilirubinemia & $61(60.4)$ & $47(62.7)$ & 0.760 \\
\hline $\begin{array}{l}\text { Transaminase } \\
\text { elevation }\end{array}$ & $65(64.4)$ & $46(61.3)$ & 0.681 \\
\hline Anemia & $30(29.7)$ & $21(28.0)$ & 0.805 \\
\hline Neutropenia & $35(34.7)$ & $22(29.3)$ & 0.456 \\
\hline Thrombocytopenia & $42(41.6)$ & $27(36.0)$ & 0.453 \\
\hline $\begin{array}{l}\text { Hand-foot } \\
\text { syndrome }\end{array}$ & $0(0.0)$ & $0(0.0)$ & NA \\
\hline Neuropathy & $1(1.3)$ & $0(0.0)$ & NA \\
\hline Bleeding & $0(0.0)$ & $0(0.0)$ & NA \\
\hline
\end{tabular}

NA - not available

activation of 5-FU in the gastrointestinal tract, it reduces the incidence of gastrointestinal toxicities [21]. S-1 showed a better anti-tumour activity and lower toxicity compared to 5-FU. A phase-I/II study suggested that S-1 was effective and had an acceptable toxicity profile in patients with advanced HCC [12]. With the deepening of the research on S-1, there were some articles published in 2015. One article aimed to compare the efficacy of S-1 versus best supportive care in advanced hepatocellular carcinoma, and demonstrated that S-1 showed an acceptable safety profile and benefit in survival in patients with advanced HCC [22]. Another article demonstrated that although S-1 did not statistically extend OS compared to the placebo in patients with sorafenib-refractory advanced HCC, the subgroup analysis showed S-1 has potential to improve OS in the clinically-important population [23].

Combined TACE and S-1: is there a better curative effect to HCC? Based on this consideration, we designed this retrospective analysis to assess the efficacy and safety of TACE plus S-1 for the treatment of BCLC Stage B HCC when they were refractory to TACE. In this analysis, a total of 612 AEs occurred during the course of the treatments, 367 in TACE plus S-1 and 245 in TACE monotherapy. There were basically no differences between the two groups except anorexia and nausea. Because of the digestive tract toxicity, we consider the differences of anorexia and nausea should be due to S-1. But anorexia and nausea could be

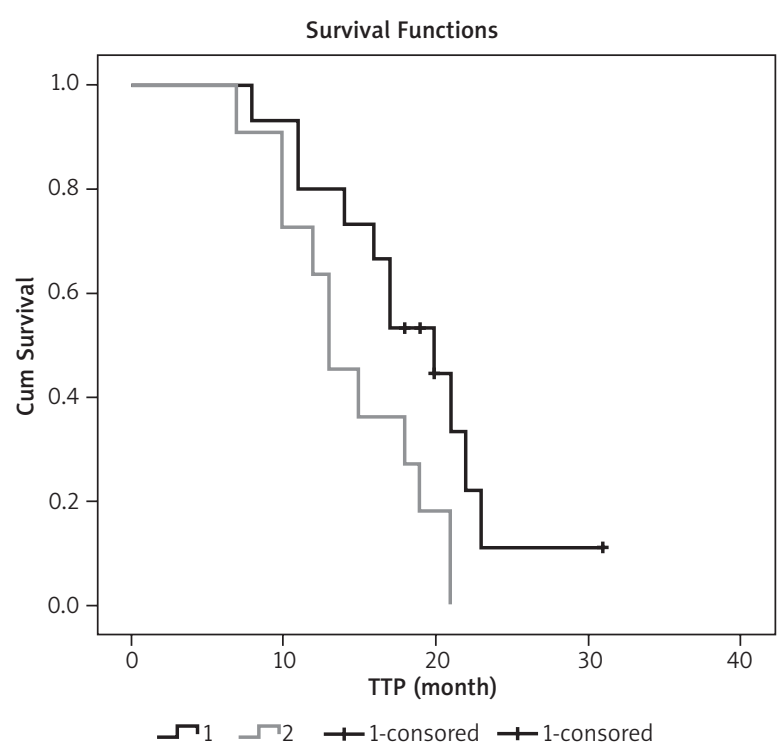

Fig. 4. Overall survival (OS) curves of TACE plus S-1 group and TACE monotherapy group

controlled by subsequent supportive therapy. Importantly, combination therapy with TACE and S-1 did not appear to lead to worse AEs than were observed in TACE monotherapy. Therefore, it was safe to use combination therapy with TACE and S-1.

There was also satisfactory TTP in this analysis. Median TTP was 6 months (range, 1-13 months) in group of TACE and S-1 versus 4 months (range, 1-5 months) in the TACE monotherapy group. And there was a significant difference between the two $(p=0.015$, $<0.05$ ). As another kind of combination therapy, a study in Asia of the combination of TACE with sorafenib in patients with HCC study (START) showed a median TTP was 415 days (approximately 13.8 months) [24]. START was a phase 2 , investigator-initiated, open-label, prospective single-arm multi-centre trial, which investigated the efficacy and safety of sorafenib combined with TACE in Southeast Asian patients with HCC, and its study population was $B C L C$ stage $B$ and A HCC. The difference was only BCLC Stage B HCC selected in the present analysis. Furthermore, the entire patients in our analysis were refractory to previous TACE. In another study, the median TTP reported for that study was 5.4 months (162 days) for the TACE plus sorafenib group compared to 3.7 months (111 days) in the placebo group [25]. Therefore, the Median TTP of TACE and S-1 of 6 months was favourable.

Different studies have shown different overall survivals (OS) in patients with BCLC stage B HCC, because of different influence factors. In the study by Bruix, median OS was 16-20 months [5]. However, the BCLC group reported a median OS of nearly 48 months for highly selected BCLC stage B HCC patients treated with DEB-TACE [26]. In our analysis, median OS of TACE plus S-1 was 18 months (95\% Cl: 15.3-24.7) while TACE monotherapy was 13 months (95\% Cl: 9.8-16.2). According to a 20-year survey on BCLC stage B HCC patients, who accepted TACE, the median OS 
was 25 months ( $\mathrm{Cl}$ : 22-28 months) with a 5-year survival of $18 \%$ [27]. It showed that TACE was an efficient option, with higher OS than for best supportive care (BSC), for selected patients with BCLC stage B HCC who were not eligible for radical treatments (for example, resection and radio-frequency ablation). Possibly based on a subclassification of BCLC stage B HCC, TACE was associated with an OS higher than expected. This indicated the need for a multidisciplinary evaluation of the BCLC stage B HCC patients to identify different prognostic subgroups, and provide individualised treatment strategies.

The DCRS of TACE plus S-1 and TACE monotherapy were $80 \%(12 / 15)$ and $63.6 \%(7 / 11)$, respectively, in this analysis. In a study of HCC patients refractory to TACE, the DCR (CD+PR+SD) was $60.4 \%$ in the sorafenib group and $28.8 \%$ in the group of hepatic arterial infusion chemotherapy (HAIC) using cisplatin group [28]. It showed when compared with HAIC using cisplatin, that sorafenib (a molecular targeted drug) demonstrated a significantly higher DCR in patients refractory to TACE $(p=0.001)$. Compared to this figure of $60.4 \%$, the DCR of $80.0 \%$ in the present analysis seemed to be higher. But the two were not in a same study, and having different baseline data, a statistical comparison was not suitable. However, the DCR of $80.0 \%$ was exciting in patients with BCLC Stage B HCC refractory to TACE. It strengthened our confidence to do further research. Differences in gene expression of hepatocellular carcinoma might be an important direction [29].

The retrospective nature and small sample size of patients included are the main limitation of our analysis. It was only small study (only 26 cases: 15 cases in TACE plus S-1 group and 11 cases in TACE monotherapy group) form single center. Statistical analysis in this case is very limited. The study group is too small to be able to draw any conclusions. We need first: a retrospective analysis of a large group from various centres and second: a prospective, more advanced trial with clearly more rigorous reporting and data monitoring or more cases from other centres.

In conclusion, TACE plus S-1 in the present analysis was tolerable and associated with an interesting TTP and OS. In the future, TACE plus S-1 may be used as a treatment method for BCLC Stage B HCC refractory to TACE.

This work was supported by the grants from Scientific Research and Innovation Foundation of Xinjiang Medical University (No. XJC2013118).

The authors declare no conflict of interest.

\section{References}

1. Parkin DM, Bray F, Ferlay J, Pisani P. Global cancer statistics, 2002. CA: a cancer journal for clinicians 2005; 55: 74-108.

2. Bruix J, Sherman M, Llovet JM, Beaugrand M, Lencioni R, Burroughs AK, et al. Clinical management of hepatocellular carcinoma. Conclusions of the Barcelona-2000 EASL conference. European Association for the Study of the Liver. J Hepatol 2001; 35: 421-30.

3. Bruix J, Sherman M. Management of hepatocellular carcinoma. Hepatology 2005; 42: 1208-36.

4. Liver EAFTSOT. EASL-EORTC clinical practice guidelines: management of hepatocellular carcinoma. J Hepatol 2012; 56: 908-43.
5. Bruix J, Sherman M, American Association for the Study of Liver D. Management of hepatocellular carcinoma: an update. Hepatology 2011; 53: 1020-2.

6. Llovet JM, Real MI, Montaña X, et al. Arterial embolisation or chemoembolisation versus symptomatic treatment in patients with unresectable hepatocellular carcinoma: a randomised controlled trial. Lancet 2002; 359: 1734-9.

7. Lo C-M, Ngan H, Tso W-K, Liu C-L, Lam C-M, Poon RT-P, et al. Randomized controlled trial of transarterial lipiodol chemoembolization for unresectable hepatocellular carcinoma. Hepatology 2002; 35: 1164-71.

8. Llovet JM, Bruix J. Systematic review of randomized trials for unresectable hepatocellular carcinoma: chemoembolization improves survival. Hepatology 2003; 37: 429-42.

9. Marelli L, Stigliano R, Triantos C, et al. Transarterial therapy for hepatocellular carcinoma: which technique is more effective? A systematic review of cohort and randomized studies. Cardiovasc Intervent Radiol 2007; 30: 6-25.

10. Llovet JM, Ricci S, Mazzaferro V, et al. Sorafenib in advanced hepatocellular carcinoma. N Eng J Med 2008; 359: 378-90.

11. Cheng A-L, Kang Y-K, Chen Z, et al. Efficacy and safety of sorafenib in patients in the Asia-Pacific region with advanced hepatocellular carcinoma: a phase III randomised, double-blind, placebo-controlled trial. Lancet Oncol 2009; 10: 25-34.

12. Furuse J, Okusaka T, Kaneko S, et al. Phase I/II study of the pharmacokinetics, safety and efficacy of $S 1$ in patients with advanced hepatocellular carcinoma. Cancer Sci 2010; 101: 2606-11.

13. Kim S-J, Han S-W, Oh D-Y, et al. Combination chemotherapy with S-1 and platinum in advanced hepatocellular carcinoma. Anticancer Res 2010; 30: 5245-50.

14. Perz JF, Armstrong GL, Farrington LA, Hutin YJ, Bell BP. The contributions of hepatitis $B$ virus and hepatitis $C$ virus infections to cirrhosis and primary liver cancer worldwide. J Hepatol 2006; 45: 529-38.

15. Altekruse SF, McGlynn KA, Reichman ME. Hepatocellular carcinoma incidence, mortality, and survival trends in the United States from 1975 to 2005. J Clin Oncol 2009; 27: 1485-91.

16. Nishikawa H, Osaki Y, Kita R, Kimura T, Ohara Y, Takeda H, et al. Comparison of transcatheter arterial chemoembolization and transcatheter arterial chemotherapy infusion for patients with intermediate-stage hepatocellular carcinoma. Oncol Rep 2014; 31: 65-72.

17. Kudo M, Izumi N, Kokudo N, Matsui O, Sakamoto M, Nakashima O, Kojiro M, Makuuchi M; HCC Expert Panel of Japan Society of Hepatology. Management of hepatocellular carcinoma in Japan: Consensus-Based Clinical Practice Guidelines proposed by the Japan Society of Hepatology (JSH) 2010 updated version. Dig Dis 2011; 29: 339-64

18. Lee JE, Bae SH, Choi JY, Yoon SK, You YK, Lee MA. Epirubicin, cisplatin, 5-FU combination chemotherapy in sorafenib-refractory metastatic hepatocellular carcinoma. World journal of gastroenterology. World J Gastroenterol 2014; 20: 235-41.

19. Lv Y, Liang R, Hu X, et al. Combination of oxaliplatin and S-1 versus sorafenib alone in patients with advanced hepatocellular carcinoma. Pharmazie 2014; 69: 759-63.

20. Ueda H, Tanaka H, Kida Y, Fukuchi H, Ichinose M. Adjuvant chemotherapy with tegafur/uracil administration after transcatheter arterial chemoembolization for advanced hepatocellular carcinoma. Oncol Rep 2008; 19: 1355-61.

21. Shirasaka T. Development history and concept of an oral anticancer agent S-1 (TS-1 $\left.{ }^{\circledR}\right)$ : its clinical usefulness and future vistas. Jpn J Clin Oncol 2009; 39: 2-15.

22. Yu P. Comparative efficacy of S-1 versus best supportive care in advanced hepatocellular carcinoma. J Clin Oncol 2015; 33 (suppl 3; abstr 466).

23. Kudo M, Moriguchi M, Numata K, Hidaka H, Tanaka H, Ikeda M, Kawazoe S, Ohkawa S, Sato Y, Okusaka T. A randomized, double-blind, placebo-controlled phase III study of S-1 in patients with sorafenib-refractory advanced hepatocellular carcinoma (S-CUBE). J Clin Oncol 2015; 33 (suppl; abstr 4018).

24. Chao Y, Chung YH, Han G, et al. The combination of transcatheter arterial chemoembolization and sorafenib is well tolerated and 
effective in Asian patients with hepatocellular carcinoma: Final results of the START trial. Int I Cancer 2015; 136: 1458-67.

25. Kudo M, Imanaka K, Chida N, et al. Phase III study of sorafenib after transarterial chemoembolisation in Japanese and Korean patients with unresectable hepatocellular carcinoma. Eur J Cancer 2011; 47: 2117-27.

26. Burrel M, Reig M, Forner A, et al. Survival of patients with hepatocellular carcinoma treated by transarterial chemoembolisation (TACE) using Drug Eluting Beads. Implications for clinical practice and trial design. J Hepatol 2012; 56: 1330-5.

27. Farinati F, Vanin V, Giacomin A, et al. BCLC stage B hepatocellular carcinoma and transcatheter arterial chemoembolization: a 20year survey by the Italian Liver Cancer group. Liver Int 2015; 35 223-31.

28. Ikeda M, Mitsunaga S, Shimizu S, et al. Efficacy of sorafenib in patients with hepatocellular carcinoma refractory to transcatheter arterial chemoembolization. J Gastroenterol 2014; 49: 932-40.

29. Li J, Huang Z, Wei L. Bioinformatics analysis of the gene expression profile of hepatocellular carcinoma: preliminary results. Contemp Oncol (Pozn) 2016; 20: 20-7.

\section{Address for correspondence}

\section{Xi-wen Fan}

Department of Intervention Radiology

the Affiliated Tumour Hospital of Xinjiang Medical University

Urumqi, Xinjiang 830011

e-mail: yishijiusheng@126.com

Submitted: 19.03 .2016

Accepted: 16.11 .2016 